\title{
BEKENSTEIN MODEL AND THE TIME VARIATION OF THE STRONG COUPLING CONSTANT
}

\author{
N. Chamoun ${ }^{1,2}$, S. J. Landau ${ }^{3 *}$, H. Vucetich ${ }^{1,3 \dagger}$, \\ ${ }^{1}$ Departamento de Física, Universidad Nacional de La Plata, \\ c.c. 67,1900 La Plata, Argentina. \\ ${ }^{2}$ Department of Physics, Higher Institute for Applied Sciences and Technology, \\ P.O. Box 31983, Damascus, Syria. \\ ${ }^{3}$ Observatorio Astronómico, Universidad Nacional de La Plata, \\ Paseo del Bosque S/N, CP 1900 La Plata, Argentina.
}

\begin{abstract}
We propose to generalize Bekenstein model for the time variation of the fine structure "constant" $\alpha_{e m}$ to QCD strong coupling constant $\alpha_{S}$. We find that, except for a "fine tuned" choice of the free parameters, the extension can not be performed trivially without being in conflict with experimental constraints and this rules out $\alpha_{S}$ variability. This is due largely to the huge numerical value of the QCD vacuum gluon condensate when compared to the matter density of the universe.
\end{abstract}

Key words: fundamental constants; elementary particles processes; cosmology

PACS: $95.30 \mathrm{Cq}, 12.38-\mathrm{t}, 98.80-\mathrm{k}$

*fellow of CONICET

${ }^{\dagger}$ member of CONICET 


\section{Introduction}

The time variation of fundamental constants may provide a connection between cosmology and particle physics. Early suggestions can be traced to Dirac [1] long ago, but many proposals leading to time-varying constants were discussed far afterwards. These can be classified to either "phenomenological" models [2, 3, 4], models providing a natural theoretical framework in terms of higher-dimensional theories like Kaluza-Klein [5, 6] and string theories [7, 8] or models based on first principles such as the Bekenstein's for $\alpha_{e m}$ variability [9]. Besides, spatial variations of the fine structure within Beckenstein's models have also been considered [10]

Actually, Bekenstein model for electromagnetism is very attractive because it is based on very general assumptions: covariance, gauge invariance, causality and time-reversal invariance of electromagnetism as well as the idea that the Planck-Wheeler length $\left(10^{-33} \mathrm{~cm}\right)$ is the shortest scale allowable in any physical theory. The very generality of its assumptions guarantee the applicability of the scheme to other "gauge" interactions such as the strong forces. Besides, it introduces a useful simplifying assumption; namely, that the gravitational sector is unaffected by the scalar field introduced to vary the coupling constant. However, it is interesting to explore first this simplified model, before a similar exploration of more general theories.

In fact, a grand unified description of electromagnetism and QCD will, presumably, predict linked time variation of low energy fundamental constants [5, 11, 12]. Nevertheless, one can anticipate that the QCD vacuum contribution, as we shall see in section 3, will be several orders of magnitud larger than the contribution coming from $\alpha_{e m}$ variability [13], so that we can assume, in the presence of a variable unifying coupling constant, that the strong forces separate cleanly from other interactions and so study them alone. To date we know of no "gauge-principled" analysis for the variability of the strong coupling constant and the object of this letter is to provide just such a study. In particular we will apply Bekenstein scheme to QCD since its assumptions are still valid for the strong interactions and find, contrary to the case of electromagnetism, that it is the vacuum, when compared to matter, which plays the dominant role as a source of variability. 


\section{Analysis}

Our starting point is the QCD Lagrangian with a varying coupling "constant"

$$
\begin{aligned}
L_{Q C D} & =L_{\epsilon}+L_{g}+L_{m} \\
& =L_{\epsilon}-\frac{1}{2} \operatorname{Tr}\left(G^{\mu \nu} G_{\mu \nu}\right)+\sum_{f} \bar{\psi}^{(f)}\left(i \gamma^{\mu} \partial_{\mu}-M_{f}+g_{0} \epsilon(x) A_{\mu} \gamma^{\mu}\right) \psi^{\left(f \gamma_{(1)}\right.}
\end{aligned}
$$

where $G^{\mu \nu}=G_{a}^{\mu \nu} t^{a}, A^{\mu}=A_{a}^{\mu} t^{a},\left[t^{a}, t^{b}\right]=i f^{a b c} t^{c}, M_{f}$ is the f-flavour quark mass and where, follwing [9], we introduced a classical scalar gaugeinvariant and dimensionless field $\epsilon(x)$. The varying coupling constant is given by $g(x)=g_{0} \epsilon(x)$ where $g_{0}$ is a constant and we require our theory governing $\epsilon$ to be scale invariant.

In order that the action be invariant under $\psi \rightarrow \psi^{\prime}=U \psi\left(U=e^{-i t^{a} \theta_{a}(x)}\right)$, we find that $\epsilon A_{\mu} \rightarrow \epsilon A_{\mu}^{\prime}=U \epsilon A_{\mu} U^{-1}-\frac{i}{g_{0}}\left(\partial_{\mu} U\right) U^{-1}$ while the gluon tensor field, transforming like $G \rightarrow G^{\prime}=U G U^{-1}$, should be given by

$$
G_{\mu \nu}^{a}=\frac{1}{\epsilon}\left[\partial_{\mu}\left(\epsilon A_{\nu}^{a}\right)-\partial_{\nu}\left(\epsilon A_{\mu}^{a}\right)+g_{0} \epsilon^{2} f^{a b c} A_{\mu}^{b} A_{\nu}^{c}\right]
$$

Similar to the electromagnetic case, it is the time reversal invariance which excludes the $G^{*} G$ term from the free gluon action while, concerning the dynamics of $\epsilon$, the same arguments in [9] apply so we take

$$
L_{\epsilon}=-\frac{1}{2} \frac{\hbar c}{l^{2}} \frac{\epsilon_{, \mu} \epsilon^{, \mu}}{\epsilon^{2}}
$$

where we merely require the scale length $l$ to be no shorter than the PlankWheeler length $L_{P}=\sqrt{\frac{\hbar G}{c^{3}}}$.

Writing the Euler-Lagrange equations for the total action $S=\int L_{Q C D} \sqrt{-g} d^{4} x$, first with respect to $A^{\mu}$, we find

$$
\left(\frac{G_{a}^{\mu \nu}}{\epsilon}\right)_{; \mu}-g_{0} f^{a b c} G_{b}^{\mu \nu} A_{\mu}^{c}+\sum_{f} g_{0} \bar{\psi} t^{a} \gamma^{\nu} \psi=0
$$

then with respect to $\epsilon$, we get

$$
\begin{aligned}
\square \ln \epsilon= & \frac{l^{2}}{\hbar c}\left[\left(G_{a}^{\mu \nu} A_{\mu}^{a}\right)_{; \nu}+g_{0} \frac{\epsilon}{2} f^{a b c} G_{a}^{\mu \nu} A_{\mu}^{b} A_{\nu}^{c}\right. \\
& \left.+\sum_{f} \bar{\psi} \frac{\partial M_{f}}{\partial \epsilon} \epsilon \psi-\sum_{f} g_{0} \epsilon \bar{\psi} A_{\mu} \gamma^{\mu} \psi\right]
\end{aligned}
$$


Substituting (4) in (5) we obtain

$$
\square \ln \epsilon=\frac{l^{2}}{\hbar c}\left[\sum_{f} \bar{\psi} \frac{\partial M_{f}}{\partial \epsilon} \epsilon \psi-\frac{1}{2} G_{a}^{\mu \nu} G_{\mu \nu}^{a}\right]
$$

This equation is analogous to the electromagnetic case but while, in electromagnetism, only matter acted as a source for both terms, by contrast, in QCD we can drop the first term working in the chiral limit $M_{f} \approx 0$ and, more importantly, we should include the vacuum contribution to the energy density in the second term. Approximating the mass in the universe by free nucleons we can write

$$
\begin{aligned}
\square \ln \epsilon & =\frac{l^{2}}{\hbar c}\left[-\frac{1}{2}\left\langle 0\left|G_{a}^{\mu \nu} G_{\mu \nu}^{a}\right| 0\right\rangle-\sum_{\text {nucleons }} \frac{\left\langle N\left|\operatorname{Tr}\left(G^{\mu \nu} G_{\mu \nu}\right)\right| N\right\rangle}{\langle N \mid N\rangle}\right] \\
& =\frac{-l^{2}}{2 \hbar c}\left[\left\langle 0\left|G_{a}^{\mu \nu} G_{\mu \nu}^{a}\right| 0\right\rangle+\rho_{m} A_{g}^{(2)}\right]
\end{aligned}
$$

where $\rho_{m}$ is the matter density of the universe and where the matrix element $A_{g}^{(2)}$ is defined for the twist-2 operator by

$$
\langle N| \operatorname{Tr}\left(G^{\mu_{1} \nu} G_{\mu_{2} \nu}\right)-\text { traces }|N\rangle=A_{g}^{(2)}\left(p^{\mu_{1}} p^{\mu_{2}}-\text { traces }\right)
$$

and has the physical meaning of the part of nucleon momentum carried by gluons.

Assuming homogeneity and isotropy for an expanding universe, and so considering only temporal variations for $\alpha_{S}$, we get

$$
\left(a^{3} \frac{\dot{\epsilon}}{\epsilon}\right)=\frac{a^{3}(t) l^{2}}{2 \hbar c} c^{2}\left[\left\langle G^{2}\right\rangle+A_{g}^{(2)} \rho_{m}\right]
$$

where $a(t)$ is the expansion scale factor. Let us consider models where the scale factor of the Universe behaves as in a flat Robertson-Walker space-time. Thus, for models with cosmological constant, we have

$$
a(t)=a\left(t_{0}\right)\left(\frac{\Omega_{m}}{\Omega_{\Lambda}}\right)^{\frac{1}{3}}\left[\sinh \frac{3}{2} \Omega_{\Lambda}^{\frac{1}{2}} H_{0} t\right]^{\frac{2}{3}}
$$

where $\Omega_{m, \Lambda}$ is the cosmological density parameter corresponding to the mass and the cosmological constant respectively and $\Omega_{m}+\Omega_{\Lambda}=1$ for flat universes. 
Models without cosmological constant are obtained in the limit $\Omega_{\Lambda} \rightarrow 0$, where the scale factor behaves as:

$$
a(t)=a\left(t_{0}\right)\left(\frac{t}{t_{0}}\right)^{\frac{2}{3}}
$$

\section{Results and Conclusion}

The matrix element $A_{g}^{(2)}$ can not be computed perturbatively and experiments give it the value of 0.48 [14] while for the other non-perturbative quantity: the gluon condensate, we can estimate it by QCD sum rules method [15]

$$
\left\langle 0\left|\frac{\alpha_{S}}{\pi} G^{2}\right| 0\right\rangle \sim(0.012 \pm 0.004) \mathrm{GeV}^{4}
$$

where the operator $G^{2}$ is renormalized at the "natural" scale $1 \mathrm{GeV}$ corresponding to the matching condition of the sum rules to avoid the appearance of large radiative corrections. In order to evaluate $\left\langle G^{2}\right\rangle$ we take $\Lambda_{Q C D}=125 \pm 25 \mathrm{MeV}$ consistent with the range of values used in QCD sum rules [16] implying, to leading $\log , \alpha_{S}(1 \mathrm{GeV})=0.336 \pm 0.0323$ and so we find

$$
\left\langle G^{2}\right\rangle \sim(0.112 \pm 0.048) \mathrm{GeV}^{4}
$$

Now since, in our model, $\rho_{m}=\frac{3 H_{0}^{2} c^{2}}{8 \pi G}\left(\frac{a\left(t_{0}\right)}{a(t)}\right)^{3}$ with $\rho_{c}=\frac{3 H_{0}^{2} c^{2}}{8 \pi G} \sim 10^{-47} \mathrm{GeV}^{4}$ we find, here, a strikingly exotic predominance of the QCD vacuum over matter and we can neglect the mass term altogether in equation (9). By integration we find then for models with cosmological constant:

$$
\begin{aligned}
\frac{\dot{\epsilon}}{\epsilon}= & \left(\frac{l}{L_{P}}\right)^{2} \frac{\left\langle G^{2}\right\rangle}{\rho_{c}} \frac{3 H_{0}^{2}}{16 \pi} \frac{\Omega_{m}}{\Omega_{\Lambda}} \frac{a_{0}^{3}}{a^{3}(t)} \times \\
& {\left[-\frac{\left(t-t_{c}\right)}{2}+\frac{\sinh \left(3 \sqrt{\Omega_{\Lambda}} H_{0} t\right)}{6 H_{0} \sqrt{\Omega_{\Lambda}}}-\frac{\sinh \left(3 \sqrt{\Omega_{\Lambda}} H_{0} t_{c}\right)}{6 H_{0} \sqrt{\Omega_{\Lambda}}}\right] }
\end{aligned}
$$

where $t_{c}$ is an unknown free parameter. In the limit of zero cosmological costant, the variation of $\epsilon$ behaves as:

$$
\frac{\dot{\epsilon}}{\epsilon}=\left(\frac{l}{L_{P}}\right)^{2} \frac{\left\langle G^{2}\right\rangle}{\rho_{c}} \frac{H_{0}^{2}}{16 \pi}\left(t-\frac{t_{c}^{3}}{t^{2}}\right)
$$


In order to evaluate equations (12) and (13) for today we need laboratory bounds on the variation of the strong coupling constant. Here, we can use a large number of data from various high energy processes ordered by increasing energy scale of the measurement as follows: $\tau$ decay, GLS sum rule, $Q \bar{Q}$ lattice, deep inelastic scattering, $R\left(e^{+}, e^{-}\right), P_{t}(w), e^{+} e^{-}$event shape and $Z$ width, giving in all a weighted average to $\Lambda_{Q C D}$ equal to $195 \pm 65 \mathrm{MeV}$ in the year 1994 [17] and $208 \pm 25 \mathrm{MeV}$ in the year 1999 [18. This allows us to take, up to leading log terms, the laboratory bound $\left|\frac{\dot{\epsilon}}{\epsilon}\right|_{\text {today }}=\frac{1}{2}\left|\frac{\alpha_{S}}{\alpha_{S}}\right|<$ $4.10^{-2} \mathrm{yr}^{-1}$.

If we assume, plausibly, $t_{c}$ of the order of $t_{0} \sim 10^{10} \mathrm{yr} \sim H_{0}^{-1}$ and take $\Omega_{\Lambda}$ in the interval $[0.25,0.75]$ with $\Omega_{m}=1-\Omega_{\Lambda}$ for models with cosmological constant (equation 10) or use equation (11) for models without cosmological constant, we find, pushing $l$ down to near its minimum allowable value $\frac{l}{L_{P}} \sim$ 1 , a constraint on $\left|t_{0}-t_{c}\right|<10^{-25} \mathrm{yr}$ which is highly strange barring a "fine tuning" situation.

For the purpose of refining the analysis, let us substitute equation (10) into (12) and integrate with $\epsilon\left(t_{0}\right)=1$ to get the following expression for the variation of $\alpha_{s}$ for models with cosmological constant

$$
\begin{aligned}
\frac{\Delta \alpha_{S}}{\alpha_{S}}= & \left(\frac{l}{L_{P}}\right)^{2} \frac{\left\langle G^{2}\right\rangle}{\rho_{c}} \frac{1}{12 \pi \Omega_{\Lambda}}\left[x \operatorname{coth} x-x_{0} \operatorname{coth} x_{0}-x_{c} \operatorname{coth} x+x_{c} \operatorname{coth} x_{0}\right. \\
& \left.+\sinh x_{c} \cosh x_{c} \operatorname{coth} x-\sinh x_{c} \cosh x_{c} \operatorname{coth} x_{0}\right]
\end{aligned}
$$

where $x=\frac{3}{2} \sqrt{\Omega_{\Lambda}} H_{0} t$ and $x_{0}\left(x_{c}\right)$ is $x$ evaluated at $t_{0}\left(t_{c}\right)$. In the limit of zero c cosmological constant, we find using equation (13)

$$
\frac{\Delta \alpha_{S}}{\alpha_{S}}=\left(\frac{l}{L_{P}}\right)^{2} \frac{\left\langle G^{2}\right\rangle}{\rho_{c}} \frac{H_{0}^{2}}{8 \pi}\left(\frac{t^{2}}{2}-\frac{t_{0}^{2}}{2}+\frac{t_{c}^{3}}{t}-\frac{t_{c}^{3}}{t_{0}}\right)
$$

Now, we can use astronomical and geophysical data giving bounds on the variation of $\alpha_{S}$ ranging over longer periods of time. In fact, the authors of [2] have derived a relation between the shift in the half-life of three long lived $\beta$ decayers $\left({ }^{187} \mathrm{Re},{ }^{40} \mathrm{~K}\right.$ and $\left.{ }^{87} \mathrm{Rb}\right)$, measured in laboratory or by comparison with the age of meteorites, and a possible temporal variation of the fundamental constants $\alpha_{e m}, \Lambda_{Q C D}$ and $G_{F}$. In this work we attribute the change 
uniquely to $\Lambda_{Q C D}$ and so we get a bound for the variation of $\alpha_{S}$ at the age of the meteorites compared to its value now $\frac{\Delta \alpha_{S}}{\alpha_{S}}=\left(0 \pm 2.1 \times 10^{-4}\right)$.

One of the most stringent limits on the time variation of fundamental constants follows from an analysis of isotope ratios of ${ }^{149} \mathrm{Sm} /{ }^{147} \mathrm{Sm}$ in the natural uranium fission reactor that took place $1.8 \times 10^{9} \mathrm{yr}$ ago at the present day site of the Oklo mine in Gabon, Africa [19, 20]. Sisterna and Vucetich [2] have shown that the information about the time variation of the strong interaction parameter $\Lambda_{Q C D}$ is very small when compared to the other fundamental constants. This is because in the chiral limit, the time variation of any strong energy difference takes the form $\frac{\Delta E}{\Delta E}=\frac{\dot{\Lambda}}{\Lambda}$; and the limits on the shift of the neutron capture resonance are not very accurate. (See also the discussion in [20].Thus, we will not include the Oklo limits in our set of data.

On the other hand, quasar absorption systems present ideal laboratories to test the temporal variation of the fundamental constants. The continuum spectrum of a quasar was formed at an epoch corresponding to the redshift $z$ of the main emission details with the relation $\lambda_{o b s}=\lambda_{l a b}(1+z)$. Knowing that the ratio of frequencies of the hyperfine $21 \mathrm{~cm}$ absorption transition of neutral hydrogen to an optical resonance transition is proportional to $x=\alpha_{e m}^{2} g_{p} \frac{m_{e}}{m_{p}}$ where $g_{p}$ is the proton $g$ factor and $m_{p}$ is its mass, we can translate a change in $x$ into a difference between the measured redshifts of the $21 \mathrm{~cm}$ and the optical absorption as follows:

$$
\frac{\Delta x}{x}=\frac{z_{o p t}-z_{21}}{(1+z)}
$$

Thus, combining the measurements of optical and radio redshifts, one can obtain bounds on $x: \frac{\Delta x}{x}=(0.7 \pm 1.1) \times 10^{-5}$ at $z=1.776$ [21], $\frac{\Delta x}{x}=$ $(0 \pm 1.2) \times 10^{-4}$ at $z=0.69$ [22] and $\frac{\Delta x}{x}=(0 \pm 2.8) \times 10^{-4}$ at $z=0.52$ [23].

Evidence for the time variation of the fine structure constant has been claimed by Webb et al [24] and further results posted recently [25, 26]. However, this detection does not suggest a power law fit. Moreover, it is found in [27] that Webb's results are in conflict with the equivalence principle when examined within Bekenstein model for $\alpha_{e m}$ variability which is in agreement with the conclusion of [13] examining all available experimental bounds in the context of typical theories predicting time variation of the fundamental constants. For the purposes of this paper,the bounds on $x$-variations will be assumed to come solely from the change of $m_{p}$ proportional to $\Lambda_{Q C D}$ and, thus, infer bounds on $\alpha_{S^{-}}$variations. Moreover, observations of molecular 
hydrogen in quasar absorption systems can be used to set bounds on the evolution of $\mu=\frac{m_{e}}{m_{p}}$ throughout cosmological time scales: $\frac{\Delta \mu}{\mu}<2 \times 10^{-4}$ at $z=2.811$ [28], and this, in turn, would imply a bound on $\alpha_{S^{-}}$-variation under a similar assumption to that for the other quasar data. Bounds on the mass ratio have also been examined [29, 30] using comparison of 21-cm hyperfine hydrogen and molecular rotatinal absorption lines. However, it is shown that this comparison actually constraints $\alpha_{e m} g_{p}$ [11.

Taking the data described above, with more "reasonable" choices for $t_{c}$, we have performed a statistical analysis using MINUIT with $H_{0}=6.64 \times$ $10^{-11} \mathrm{yr}^{-1}$ and obtained the results in the table 1 for the free parameter in the

model $\frac{l}{L_{p}}$ with $99 \%$ of confidence level. Furthermore, we could check that, as long as $t_{c}$ is far from the unnatural "fine tuning" situation, we always get $l$ shorter than $L_{P}$ by too many orders of magnitude in clear conflict with the postulates adopted in the framework. While integrating, we have assumed the value of the gluon condensate $\left\langle G^{2}>\right.$ constant whereas it might vary over cosmological time scales. However, we do not expect such variations to be large enough to change the conclusion above. Even though length scales shorter than the Planck-Wheeler length $L_{P}$ might enter physics in the context of "new" theories, namely superstrings, it is extremely unlikely that our tiny $l$ could be accommodated so as to recover the axioms of the Bekenstein model. Since the assumptions of this model are reasonable, comparison with experiments, excludes any direct generalization of the model to QCD and consequently rules out $\alpha_{S}$ variability in accordance with the strong principle of equivalence. Although it is possible that more general schemes, involving more realistic assumptions on the structure of the $\epsilon$ field or its interactions with gravitation, may resist comparison with the experiment, the present study shows that it will be difficult to build them from first principles without some fine tunning mechanism.

\section{Acknowledgements}

This work was supported in part by CONICET, Argentina. N. C. recognizes economic support from the Third World Academy of Science (TWAS).

\section{References}


Table 1: Constraints on $\frac{l}{L_{p}}$ using the full data set of bounds on $\frac{\Delta \alpha_{s}}{\alpha_{s}}$ for flat models $\left(\Omega_{\Lambda}+\Omega_{m}=1\right)$ with different cosmological constant. $t_{c}=\gamma t_{0}$

\begin{tabular}{lll}
\hline$\Omega_{\Lambda}$ & $\gamma$ & $\frac{l}{L_{p}}$ \\
\hline 0.75 & 0.3 & $(0 \pm 5) \times 10^{-25}$ \\
0.75 & 0.7 & $(1 \pm 4) \times 10^{-25}$ \\
0.5 & 0.3 & $(0 \pm 6) \times 10^{-25}$ \\
0.5 & 0.7 & $(2 \pm 7) \times 10^{-25}$ \\
0.25 & 0.3 & $(0 \pm 7) \times 10^{-25}$ \\
0.25 & 0.7 & $(4 \pm 8) \times 10^{-25}$ \\
0 & 0.3 & $(0 \pm 7) \times 10^{-25}$ \\
0 & 0.7 & $(0 \pm 2) \times 10^{-24}$
\end{tabular}

[1] P. A. M. Dirac, Nature (London) 139, 323 (1937), and Proc. Roy. Soc. London, Ser. A 165, 199 (1938)

[2] P. Sisterna and H. Vucetich, Phys. Rev. D 41 4, 1034 (1990)

[3] P. Sisterna and H. Vucetich, Phys. Rev. D 44 10, 3096 (1991)

[4] A. Albrecht and J. Magueijo, Phys. Rev. D 59 04, 3516 (1999)

[5] W. J. Marciano, Phys. Rev. Lett. 52 7, 489 (1984)

[6] J. Griego and H. Vucetich, Phys. Rev. , D 40 6, 1904 (1989)

[7] Y. Wu and Z. Wang, Phys. Rev. Lett., 57 16, 1978 (1986)

[8] K. Maeda, Mod. Phys. Lett. , A 3 3, 243 (1988)

[9] J. D. Bekenstein, Phys. Rev. , D 25 6, 1527 (1982)

[10] J. D. Barrow and C. O'Toole, astro-ph/9904116

[11] M. J. Drinkwater, J. K. Webb, J. D. Barrow and V. V. Flambaum, Mon. Not. R. Astron. Soc. 295, 457 (1998).

[12] A.B.Campbell and K.A.Olive Phys.Lett. B345, 429 (1995) 
[13] S. Landau and H. Vucetich astro-ph/0005316

[14] F. J. Yndurain, The Theory of Quarks and Gluon Interactions, 3rd ed, Springer, Berlin (1999)

[15] Vacuum Structure and QCD Sum Rules, ed. M. A. Shifman, NorthHolland (1992)

[16] G. G. Ross and N. Chamoun, Phys. Lett., B 380, 151 (1996)

[17] Particle Physics Booklet, AIP (1994)

[18] Particle Data Group, http://pdg.lbl.gov/ (June 14, 2000)

[19] A. I. Schlyakter, Nature 25, 340 (1976)

[20] T. Damour and E. Dyson, Nucl. Phys. B 480 (1996) 37

[21] L. L. Cowie and A. Songaila, Ap. J., 453, 596 (1995)

[22] A. M. Wolfe and M. M. Davis, Astron. J., 84699 (1979)

[23] A. M. Wolfe, R. Brown and M. Roberts, Phys. Rev. Lett., 37, 179 (1976)

[24] J. K. Webb, V. V. Flambaum, C. W. Churchill, M. J. Drinkwater, and J. D. Barrow, Phys. Rev. Lett. 82, 884 (1999).

[25] M. T. Murphy, J. K. Webb, V. V. Flambaum, V. A. Dzuba, C. W. Churchill, J. X. Prochaska, J. D. Barrow, A.M. Wolfe astro-ph/0012419

[26] J.K. Webb, M.T. Murphy, V.V. Flambaum, V.A. Dzuba, J.D. Barrow, C.W. Churchill, J.X. Prochaska, A.M. Wolfe, astro-ph/0012539

[27] M.Livio and M. Stiavelli Ap.J.Lett. 507 L135 (1998)

[28] A. Y. Pothekin et al., Ap. J. 505, 523 (1998)

[29] B. E. J. Pagel Phil.Trans.Roy.Soc. A310 245 (1983)

[30] D. A. Varshalovich and A. Y. Pothekin Astron.Lett 22 1, (1996) 